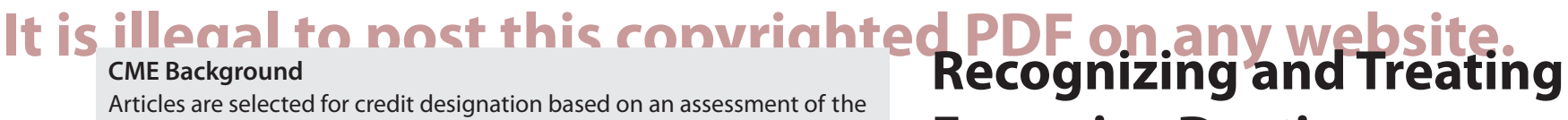 educational needs of CME participants, with the purpose of providing readers with a curriculum of CME articles on a variety of topics throughout each volume. Activities are planned using a process that links identified needs with desired results. \\ To obtain credit, read the article, correctly answer the questions in the Posttest, and complete the Evaluation. \\ Excessive Daytime Sleepiness in Patients With Narcolepsy
}

This ACADEMIC HIGHLIGHTS section of The Journal of Clinical Psychiatry presents the highlights of the teleconference series "Recognizing and Treating Excessive Daytime Sleepiness in Patients With Narcolepsy," which was held in February and April 2020. This report was prepared and independently developed by the CME Institute of Physicians Postgraduate Press, Inc., and was supported by an educational grant from Harmony Biosciences, LLC.

The teleconference was chaired by Thomas Roth, PhD, Henry Ford Hospital Sleep Center and Department of Psychiatry, Wayne State University School of Medicine, Detroit, and Department of Psychiatry, University of Michigan College of Medicine, Ann Arbor. The faculty was John W. Winkelman, MD, PhD, Sleep Disorders Clinical Research Program and Departments of Psychiatry and Neurology, Massachusetts General Hospital; and Department of Psychiatry, Harvard Medical School, Boston.

\section{CME Objective}

After studying this article, you should be able to:

- Use evidence-driven strategies for diagnosing and treating excessive daytime sleepiness and narcolepsy

\section{Accreditation Statement}

The CME Institute of Physicians Postgraduate Press, Inc., is accredited by the Accreditation Council for Continuing Medical Education to provide continuing medical education for physicians.

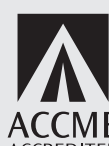
ACCME

\section{Release, Expiration, and Review Dates}

This educational activity was published in November 2020 and is eligible for AMA PRA Category 1 Credit $^{\mathrm{TM}}$ through December 31, 2022. The latest review of this material was November 2020.

\section{Financial Disclosure}

All individuals in a position to influence the content of this activity were asked to complete a statement regarding all relevant personal financial relationships between themselves or their spouse/partner and any commercial interest. The CME Institute has resolved any conflicts of interest that were identified. In the past year, Marlene P. Freeman, MD, Editor in Chief, has received research funding from JayMac and Sage; has been a member of the advisory boards for Otsuka, Alkermes, and Sunovion; has been a member of the Independent Data Safety and Monitoring Committee for Janssen; has been a member of the Steering Committee for Educational Activities for Medscape; and, as a Massachusetts General Hospital (MGH) employee, works with the MGH National Pregnancy Registry, which is sponsored by Teva, Alkermes, Otsuka, Actavis, and Sunovion, and works with the MGH Clinical Trials Network and Institute, which receives research funding from multiple pharmaceutical companies and the National Institute of Mental Health. No member of the CME Institute staff reported any relevant personal financial relationships. Faculty financial disclosure appears on the next page.

\section{J Clin Psychiatry 2020;81(6):HB19045AH3C}

To cite: Roth T, Winkelman JW. Recognizing and treating excessive daytime sleepiness in patients with narcolepsy. J Clin Psychiatry. 2020;81(6):HB19045AH3C.

To share: https://doi.org/10.4088/JCP.HB19045AH3C

(c) Copyright 2020 Physicians Postgraduate Press, Inc.

Thomas Roth, $\mathrm{PhD}$, and John W. Winkelman, MD, PhD

들 xcessive daytime sleepiness (EDS) is described as inappropriate and undesirable sleepiness during waking hours. ${ }^{1}$ Daytime sleepiness is prominent in US adults, with about one-third reporting EDS. ${ }^{2}$ Quality of life is reduced in individuals with EDS, and EDS is associated with compromised cognitive abilities and work productivity. ${ }^{3}$ Both EDS and fatigue are common symptoms among patients in primary care and in specialty medicine settings. ${ }^{4}$

Individuals with EDS may have a sleep disorder such as narcolepsy. Narcolepsy can occur with or without cataplexy (a sudden loss of muscle tone associated with strong emotion). ${ }^{5}$ The International Classification of Sleep Disorders, Third Edition ${ }^{6}$ (ICSD-3) categorizes narcolepsy with and without cataplexy as narcolepsy type 1 and narcolepsy type 2 , respectively. Untreated or insufficiently managed narcolepsy is associated with significant psychosocial disability. ${ }^{7}$ Fortunately, various treatment modalities are efficacious in the treatment of narcolepsy with and without cataplexy.

This report, based on presentations given by Thomas Roth, PhD, and John W. Winkelman, MD, $\mathrm{PhD}$, will address how to screen patients for EDS, diagnose narcolepsy, select evidence-based treatments for EDS, and monitor for residual EDS in patients being treated for narcolepsy.

\section{SCREENING FOR EXCESSIVE DAYTIME SLEEPINESS AND DIAGNOSING NARCOLEPSY}

Dr Winkelman began with a discussion about the prevalence of narcolepsy, which affects about 1 in 2,000 people. ${ }^{8}$ It is a lifelong condition, he stated, with symptoms often beginning in childhood, adolescence, or young adulthood (ages 7 to 25 years), although they can occur later. ${ }^{9}$ Unfortunately, individuals with narcolepsy frequently encounter a long delay from symptom onset to the time of diagnosis. ${ }^{10}$ For individuals who have symptom onset before the age of 18 years, two-thirds have a diagnostic delay of greater than 5 years, and nearly half have a diagnostic delay of more than 10 years. ${ }^{10}$ 


\section{It is illenal to nost this Credit Designation}

The CME Institute of Physicians Postgraduate Press, Inc., designates this journal-based CME activity for a maximum of 1 AMA PRA Category 1 Credit $^{\mathrm{TM}}$. Physicians should claim only the credit commensurate with the extent of their participation in the activity.

Note: The American Academy of Physician Assistants (AAPA) accepts certificates of participation for educational activities certified for AMA PRA Category $1 \mathrm{Credit}^{\mathrm{TM}}$ from organizations accredited by ACCME or a recognized state medical society. Physician assistants may receive a maximum of 1 hour of Category I credit for completing this program.

\section{Financial Disclosure}

Dr Roth is a consultant for and has received honoraria from Merck, Idorsia, Eisai, Alarion, Takeda, Jazz, Click Therapeutics, Avadel, GlaxoSmithKline, and SEQ; and is a member of the speaker/advisory board for Eisai. Dr Winkelman is a consultant for Avadel and CVS; has received grant/research support from Merck and RLS Foundation; is a member of the speaker/advisor board for Massachusetts General Hospital Psychiatry Academy; and has received financial or material support from Winston and Strawn and UpToDate.

\section{Review Process}

The faculty member(s) agreed to provide a balanced and evidence-based presentation and discussed the topic(s) and CME objective(s) during the planning sessions. The faculty's submitted content was validated by CME Institute staff, and the activity was evaluated for accuracy, use of evidence, and fair balance by the Chair and a peer reviewer who is without conflict of interest.

The opinions expressed herein are those of the faculty and do not necessarily reflect the opinions of the CME provider and publisher or the supporter.

\section{Patient Perspectives}

When the diagnosis of narcolepsy is delayed, individuals can experience substantial self-doubt, as the following comment illustrates:

"I had always been so engaging and upbeat, but I constantly felt physically drained, and no matter how much I slept, I would always feel just as drained. I could sleep 16 hours and still need 3 naps during the day to feel okay. I felt like an idiot, a lazy sack of nothing useful to nobody, someone who was never going to be able to do anything with my life because I would never be awake enough to do it."11

\section{Impact of Narcolepsy}

Narcolepsy is associated with problems in school and work performance, ${ }^{10}$ including increased educational difficulties, high rates of unemployment, and impairments in attention and executive functioning tasks that involve higher demands on inhibition or task-management abilities.
Figure 1. Activity Limitations Reported by Individuals With Narcolepsy $(\mathrm{N}=2,017)^{\mathrm{a}}$

"Are there specific activities that are important to you but that you cannot do at all or as fully as you would like because of your condition?"

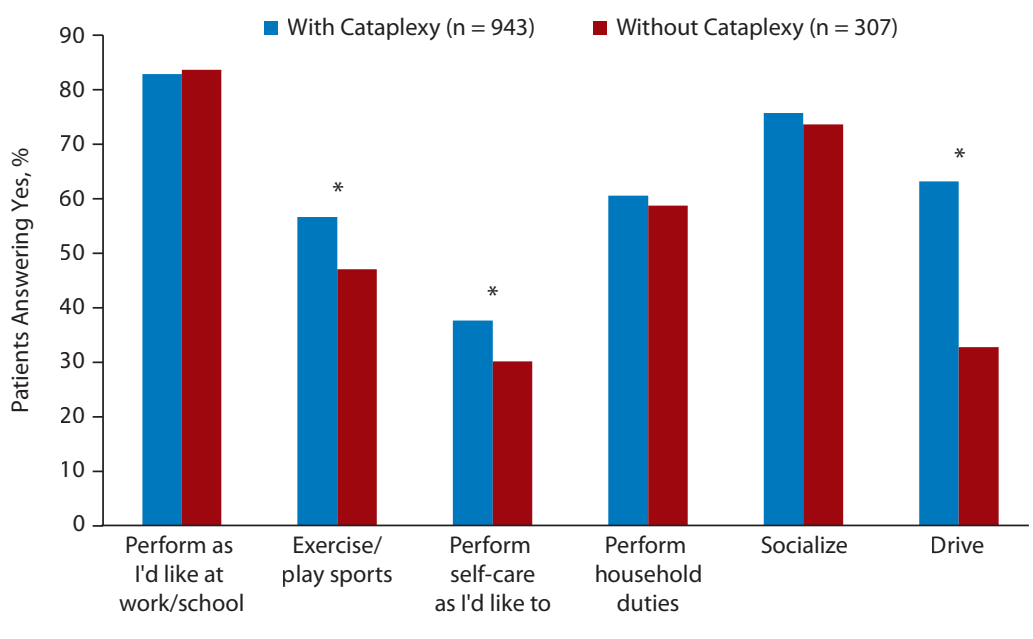

Activities

${ }^{\mathrm{a}}$ Data from Maski et al. ${ }^{10}$

$* P<.05$.

Quality of life. Dr Winkelman presented studies demonstrating that people with narcolepsy have reduced quality of life and health-related quality of life. ${ }^{12,13}$ In a survey of 49 patients with narcolepsy, the majority reported having lost or left a job (52\%), falling asleep at work (67\%), having relationship problems (56\%), and struggling to meet friends (59\%). ${ }^{14}$ A large survey ${ }^{10}$ that asked patients about specific activities reported that a substantial proportion experience limitations due to their symptoms (Figure 1).

In children and adolescents, narcolepsy is associated with impaired school performance. ${ }^{12}$ Students experience poor concentration and memory deficits, and they get disciplined for falling asleep, which can stigmatize them as being lazy, unmotivated, or on drugs. They have difficulty with athletics and other after-school activities, and they may be bullied. In a study ${ }^{15}$ of children with narcolepsy (mean age 11.6 years), $30.4 \%$ had problems with school absenteeism compared with $8.9 \%$ of control youths (mean age 13.5 years), and $28.6 \%$ had repeated a grade level compared with $7.6 \%$ of controls ( $P=.002$ for both).

Comorbidities. People with narcolepsy often have comorbid disorders, particularly psychiatric illnesses. In a study ${ }^{16}$ comparing a communitybased sample of individuals with narcolepsy $(n=68)$ versus a control group $(n=272)$, those with narcolepsy had more psychiatric and medical disorders both at diagnosis and at follow-up almost 10 years later. Conditions that were more common among individuals with narcolepsy versus the control group were endocrine disorders, depression, obstructive sleep apnea, chronic low back pain, and obesity. ${ }^{16}$

Another study ${ }^{17}$ comparing participants with narcolepsy $(n=320)$ versus a control group $(n=1,464)$ found high rates of psychiatric disorders in the narcolepsy group. The most frequent conditions among the narcolepsy group were major depressive disorder (MDD; odds ratio $[\mathrm{OR}]=2.67)$ and social anxiety disorder $(\mathrm{OR}=2.43)$, which both affected nearly $20 \%$ of patients. The occurrences of bipolar disorders $(\mathrm{OR}=4.56)$, panic disorder $(\mathrm{OR}=3.23)$, and posttraumatic stress disorder $(\mathrm{OR}=2.11)$ were also increased in the narcolepsy group. While narcolepsy is not 
central nervous system disturbances.

\section{Screening for Sleep Disorders}

Dr Winkelman shared that, in his clinical experience, patients often present with 1 or more of the following symptoms when they report feeling "tired": sleepiness, fatigue, and apathy. Despite some overlap, these symptoms have different features. A patient with EDS may report difficulty staying awake while reading, resting, or watching television, while a patient with fatigue may express feelings of apathy or listlessness rather than sleepiness. ${ }^{3}$

\section{Patient Perspectives}

Individuals with narcolepsy describe the effects of their illness in the following ways:

"For years and years, I thought I wasn't doing life right. I was sleeping much longer at night than any of my friends, but I still couldn't stay awake during the day. I couldn't study properly. I couldn't socialize. I felt like I was failing as a person."18

"By the end of my sophomore year, I was sleeping through every class, coming home and falling asleep exhausted, and waking up to do the cycle all over again. In addition, I often had difficulty sleeping at night because I had nightmares that I was paralyzed and could not move. I was extremely depressed and could barely function."19

In patients whose primary complaint is EDS, the first step is to assess the extent of their daytime sleepiness with the Epworth Sleepiness Scale (ESS).$^{20}$ The ESS is a self-administered questionnaire in which individuals are asked to rate, on a 4-point scale (0-3), their usual chances of falling asleep while engaged in 8 activities. Patients can complete the ESS in only a few minutes, and a total score of 10 or higher indicates the need for further evaluation.

Dr Winkelman advised clinicians to ask questions about medications and environmental factors that could be interfering with sleep. Next, he recommended that clinicians ask patients to track how much time they are spending in bed. Monitoring is usually done with a sleep diary or actigraphy. In a sleep diary, patients record information about their sleeping habits, while actigraphy involves patients wearing an actigraph or actiwatch (now widely available, eg, Apple Watch, Fitbit), which continuously measures limb movement and light intensity to determine periods of sleep and wake. ${ }^{21}$ Patients should fill out sleep diaries for 2 weeks, with the goal of spending at least 8 hours in bed per night. Actigraphy or sleep diaries should be performed prior to sleep studies to rule out chronic sleep deprivation, abnormal phase of the circadian cycle, and shift work disorder. ${ }^{22}$

If patients are getting an adequate amount of time in bed and adequate sleep according to their sleep diary and still complain of daytime sleepiness, clinicians must determine through a sleep laboratory evaluation if there is adequate sleep quality or whether impairments exist, such as obstructive sleep apnea (OSA), periodic limb movement disorder, or pharmacologic or environmental disturbances. ${ }^{23}$ If the sleep laboratory assessment results convey that patients are getting sleep that is of adequate quality, then their EDS may be caused by medical, neurologic, or psychiatric conditions or a sleep-wake disorder such as OSA or narcolepsy.

\section{Tests for Narcolepsy}

Two tests, performed in a sleep disorders clinic, are used to establish a diagnosis of narcolepsy. Dr Winkelman explained that an overnight stay for a polysomnogram (PSG) is typically followed the next day by the Multiple Sleep Latency Test (MSLT). ${ }^{24}$

Polysomnogram. The PSG is an overnight recording of brain and muscle activity, breathing, and eye movements. A PSG can help reveal whether rapid eye movement (REM) sleep occurs early in the sleep cycle and if an individual's symptoms result from another sleep disorder, such as OSA. ${ }^{9}$

Multiple Sleep Latency Test. The MSLT is an objective test for EDS in people with narcolepsy and idiopathic hypersomnia. ${ }^{25}$ The MSLT is done the day after the PSG to ensure that the prior night's sleep is adequate. Beginning within 3 hours after awakening, Dr Winkelman explained, individuals are given 5 nap opportunities at 2-hour intervals. If they do not fall asleep within 20 minutes, that nap opportunity is ended. If they do fall asleep, they have 15 minutes to sleep, and then they are awakened.

The key endpoints on the MSLT are the average time to fall asleep and whether any of the naps contained REM sleep. Individuals with narcolepsy tend to fall asleep quickly (ie, in less than 8 minutes on average across the MSLT naps) and enter REM sleep during 2 or more naps. Thus, the MSLT results can highlight 2 major indicators of narcolepsy: (1) falling asleep quickly even after a good night's sleep; and (2) impaired regulation of REM sleep. In contrast, individuals without narcolepsy take much longer to fall asleep during naps and will rarely enter REM sleep during a nap. ${ }^{26}$

Dr Winkelman recommended that in the week prior to the MSLT, individuals should keep a sleep $\log { }^{25}$ This log will show if sleep duration was adequate leading up to the test. Additionally, in the weeks prior to taking the MSLT, individuals should discontinue medications that suppress REM sleep, such as serotonergic antidepressants, and medications that produce alertness or excessive sleepiness, such as stimulants and nocturnal sedatives, if possible. If patients are unable to discontinue these medications, these agents will complicate the interpretation of the MSLT, Dr Winkelman added. However, if there is concern about a return of depression with antidepressant discontinuation, for example, then the MSLT interpretation should indicate that such medications were being used.

Other tests. Due to the connection between narcolepsy and a deficiency in hypocretin (also known as orexin), the sleep-regulating brain chemical, clinicians may consider using the cerebrospinal fluid (CSF) hypocretin-1 test. In this test, patients undergo a lumbar puncture for a sample 
narcolepsy, while values above $200 \mathrm{pg} / \mathrm{mL}$ are considered normal. The CSF hypocretin-1 results must be interpreted within the clinical context and may be most helpful in cases with cataplexy and when the MSLT is difficult to interpret. $^{28}$

(For information on other tests, see the online activity "Screening for Excessive Daytime Sleepiness and Diagnosing Narcolepsy" in this CME series.)

\section{Differential Diagnosis of Central Hypersomnias}

The MSLT and CSF-hypocretin-1 provide helpful information to differentiate 3 central hypersomnias: narcolepsy type 1 , narcolepsy type 2 , and idiopathic hypersomnia. ${ }^{6,22}$

Narcolepsy. In the ICSD $-3,{ }^{6}$ narcolepsy type 1 is defined as the presence of EDS with either cataplexy and a positive MSLT (mean sleep latency time of $\leq 8$ minutes and $\geq 2$ sleep-onset REM periods) or CSF hypocretin deficiency. Individuals with narcolepsy type 1 have disturbed sleepwake state control with difficulty staying awake during the day and staying asleep at night. They also have REM sleep dysregulation, which is a breakthrough of REM sleep into wakefulness or into the boundary between wake and sleep, and this dysregulation is manifested by cataplexy, sleep paralysis, and hypnagogic and hypnopompic hallucinations. ${ }^{6}$ Cataplexy is sudden loss of muscle tone in response to a strong emotion, such as laughter, sadness, and anger.

Among the central hypersomnias, narcolepsy type 1 is the best understood with its association to human leukocyte antigen subtypes, specifically DQB1*0602 and DR2/DRB1*1501, and decreased CSF hypocretin. ${ }^{8,9,29}$

Narcolepsy type 2 is also characterized by difficulties with sleep-wake control-most prominently, EDS—but without cataplexy. ${ }^{6,9}$ Diagnosis can be more challenging than with narcolepsy type 1, Dr Winkelman noted, because patients do not have obvious symptoms like cataplexy and their symptoms may change over time. He recommended that clinicians use a detailed clinical history (to rule out other causes of EDS), PSG, and MSLT to confirm a diagnosis of narcolepsy type $2 .{ }^{25}$ Narcolepsy type 2 requires a mean MSLT sleep onset latency $<8$ minutes and 2 sleep-onset REM episodes during the MSLT.

Idiopathic hypersomnia. The etiology of central hypersomnia is poorly understood. ${ }^{30}$ Individuals with this disorder have EDS and/or prolonged nighttime total sleep times-more than 11 hours of sleep on a habitual basis. They do not have the REM sleep dysregulation that is present in narcolepsy type 1 , such as cataplexy and sleep paralysis. ${ }^{6}$ Dr Winkelman identified the following other features of idiopathic hypersomnia that distinguish it from narcolepsy: the absence of multiple REM naps on the MSLT, the presence of long habitual sleep periods and naps, a feeling of grogginess upon awakening (whereas patients with narcolepsy typically feel refreshed after naps), and high sleep efficiency on the PSG. ${ }^{22}$

\section{Case Practice Question}

Discussion of the best response can be found at the end of the activity.

Case 1. Katie is a 25-year-old patient who presents with a 3-year history of increasing excessive daytime sleepiness (her current ESS score is 15). This problem began after she developed panic disorder, for which she is receiving treatment with mirtazapine and clonazepam. Katie has been putting on weight, saying that she's too tired to exercise. Which piece of information below would be least helpful to consider in making a diagnosis of Katie's excessive sleepiness?

a. Usual bedtime and waketime

b. Body mass index and presence of snoring and witnessed apneas

c. Cholesterol level

d. Presence of cataplexy, sleep paralysis, and hypnagogic hallucinations

\section{TREATING EDS IN PATIENTS WITH NARCOLEPSY}

As illustrated by the patient remarks below, Dr Roth described people with EDS as having an irrepressible need to sleep and experiencing unintended lapses in vigilance during times when they need to be alert. ${ }^{6,31}$

\section{Patient Perspectives}

"It's different from how people imagine it, though (ie, instantly falling asleep face-first into a bowl of soup or something). It's more like overwhelmingly bad jet lag. Like you can't possibly stay awake, no matter how hard you try."18

"It is so painful to stay awake sometimes that my body actually hurts. It is struggling to do the housework, make the meals, stay ahead of the piles of laundry. It is missing appointments, being late to meetings, forgetting chores. It is being unable to drive and relying on others to give you and your children rides to events.... And it is the isolation. You only go out when you have to. Even walking brings its own set of risks."132

The American Academy of Sleep Medicine (AASM) released practice parameters for narcolepsy in 2000 and provided a 2007 update, ${ }^{33}$ which summarized treatments based on evidence classification. ${ }^{34,35}$ Newly revised 2020 practice guidelines* have been drafted. The AASM recommendations, as well as treatment options not included in the AASM parameters, are discussed below, although the 2020 guidelines are subject to change.

Modafinil. Modafinil is approved by the US Food and Drug Administration (FDA) at $200 \mathrm{mg} / \mathrm{d}$ for the treatment of narcolepsy in adults and is indicated to improve wakefulness in patients with excessive sleepiness. ${ }^{36}$ The R-enantiomer of modafinil, armodafinil, has a longer half-life and is indicated for narcolepsy-related excessive sleepiness in adults at $150-250 \mathrm{mg} / \mathrm{d} .{ }^{37}$ The AASM

*https://aasm.org/clinical-resources/practice-standards/ practice-guidelines/ 
for EDS in $2000^{38}$ and again in $2007^{33}$ on the basis of multiple level I and level II studies. The 2020 draft AASM guidelines* provide a strong recommendation for the use of modafinil for narcolepsy. Research has demonstrated that modafinil reduces EDS vs placebo at daily dosages of $300 \mathrm{mg}^{39}$ and $200 \mathrm{mg}$ and $400 \mathrm{mg},{ }^{40-42}$ and Broughton et $\mathrm{al}^{40}$ found no significant difference between doses on EDS reduction.

Dr Roth discussed a long-term study ${ }^{43}$ of 478 patients diagnosed with narcolepsy, $75 \%$ of whom received $400 \mathrm{mg} / \mathrm{d}$ of modafinil. Researchers found an improvement in disease severity in more than $80 \%$ of patients and a significant improvement in ESS scores over the 40 -week trial. ${ }^{43}$ No evidence of the development of tolerance to the medication was found.

A split-dose regimen of modafinil may be appropriate for some patients. In a randomized, double-blind study of 32 patients, ${ }^{44} 400 \mathrm{mg} / \mathrm{d}$ of modafinil administered in split doses achieved improved evening wakefulness compared to oncedaily 200-mg and 400-mg doses. Modafinil is not associated with a significant potential for abuse ${ }^{45}$ and generally has a minimal occurrence of adverse events ${ }^{46}$ that range from mild to moderate in severity. ${ }^{43}$

Sodium oxybate. Sodium oxybate is FDA-approved for the treatment of narcolepsy-related EDS and cataplexy. The medication is taken orally in a nightly split-dose regimen (half at bedtime and half 4 hours later). It is initiated at $4.5 \mathrm{~g}$ per night and titrated to therapeutic effect, with a total recommended dose in the range of 6-9 g. ${ }^{47}$ The 2007 AASM practice parameters recommended sodium oxybate as a first-line treatment option, ${ }^{33}$ as level I studies found that it reduced ESS scores for patients with narcolepsy. The 2020 draft AASM guidelines* provide a strong recommendation for the use of sodium oxybate for narcolepsy. Dr Roth pointed out that sodium oxybate has abuse potential that should be considered, but the risk is low in patients with narcolepsy using a therapeutic dose. ${ }^{46}$ The agent is generally safe when properly dosed, but because the potential risk for teratogenicity in humans has not been studied, sodium oxybate is not recommended during pregnancy. Patients should avoid the use of alcohol and sedative hypnotics when taking sodium oxybate, Dr Roth added.

Amphetamines and methylphenidate. Amphetamine and methylphenidate are FDA-approved for the treatment of narcolepsy. ${ }^{33}$ In the 2000 AASM recommendations ${ }^{38}$ for EDS, level II and level V studies supported the use of amphetamine, methamphetamine, and dextroamphetamine at doses ranging from $15 \mathrm{mg} / \mathrm{d}$ to $60 \mathrm{mg} / \mathrm{d}$ and methylphenidate at doses from $10 \mathrm{mg} / \mathrm{d}$ to $60 \mathrm{mg} / \mathrm{d}$ as second-line treatment; the recommendation was repeated in the 2007 AASM update. ${ }^{33}$ The 2020 draft AASM guidelines* provide conditional recommendations for the use of methylphenidate and dextroamphetamine for narcolepsy. The available studies are not sufficiently powered to

*https://aasm.org/clinical-resources/practice-standards/practice-guidelines/ provide a comprehensive understanding of the benefit-torisk ratio for these treatments. ${ }^{33}$ Evidence indicates that tolerance-but not addiction-to amphetamines ${ }^{48,49}$ and methylphenidate ${ }^{46}$ may be a problem for patients with narcolepsy.

Polytherapy. The 2007 AASM practice parameters included combination therapy, consisting of 1 short-acting and 1 long-acting psychostimulant, rated as a third-line treatment option. ${ }^{33}$ This approach may achieve alertness quickly and maintain it for longer periods compared to monotherapy, while potentially avoiding the development of insomnia. ${ }^{38}$

Combining pharmacologic agents with different mechanisms of action is not a common approach for narcolepsy, and polytherapy with modafinil and sodium oxybate is not mentioned in the AASM treatment parameters.

Selegiline. Selegiline is a monoamine oxidase inhibitor indicated for Parkinson disease and major depressive disorder and is sometimes used off-label for narcolepsy; some evidence indicates efficacy for selegiline in reducing EDS. ${ }^{50,51}$ The 2000 AASM practice parameters recommended selegiline as a second-line treatment option for addressing both EDS and cataplexy, while cautioning that the medication was expensive and that effective dosing was associated with an increased risk for diet-induced hypertension. ${ }^{38}$ The 2007 AASM treatment parameters downgraded selegiline to a third-line option, citing lack of clinical experience in using the agent to treat narcolepsy, as well as drug and diet interactions. ${ }^{33}$ In the 2020 draft AASM guidelines, ${ }^{*}$ selegiline is not listed as a recommended option due to insufficient/inconclusive evidence.

Solriamfetol. Dr Roth identified solriamfetol as a dopamine-norepinephrine reuptake inhibitor that is FDA-approved for EDS associated with narcolepsy or obstructive sleep apnea. ${ }^{52}$ A double-blind, randomized, placebo-controlled study ${ }^{53}$ found that solriamfetol at $150 \mathrm{mg} / \mathrm{d}$ and $300 \mathrm{mg} / \mathrm{d}$ was effective at reducing sleepiness and improving wakefulness over 12 weeks as measured by ESS and Maintenance of Wakefulness Test (MWT) scores. Solriamfetol has a rapid uptake (reaching peak serum concentration in about 2 hours), can be taken with or without food, ${ }^{52}$ and has been found to be generally safe, without serious or severe treatment-emergent adverse events. ${ }^{53,54}$ However, solriamfetol is associated with abuse potential similar to or lower than that of phentermine. ${ }^{54}$ The 2020 draft AASM guidelines* provide a strong recommendation for the use of solriamfetol for narcolepsy.

Pitolisant. Pitolisant, an inverse histamine-3-receptor agonist, is the first non-controlled substance approved by the FDA to treat narcolepsy ${ }^{55}$ and is indicated for EDS in adults. ${ }^{55}$ The recommended dose range for this oncedaily oral medication is $17.8 \mathrm{mg}$ to $35.6 \mathrm{mg}$, initiated on a titration schedule starting at $8.9 \mathrm{mg} .{ }^{55}$ Pitolisant has a favorable tolerability profile when compared with placebo and active comparators. ${ }^{56}$ Additionally, pitolisant has demonstrated efficacy in treating cataplexy. ${ }^{57}$ 
that pitolisant is not inferior to modafinil in relieving EDS and is superior in the treatment of cataplexy, meaning that the agents perform equally well for narcolepsy type 2 but that pitolisant would be more effective for narcolepsy type 1 . A prior network metaanalysis $^{59}$ compared modafinil, pitolisant, and sodium oxybate; results indicated that modafinil (200-400 mg/d), sodium oxybate $(9 \mathrm{~g} / \mathrm{d}$ ), and pitolisant (up to $40 \mathrm{mg} / \mathrm{d}$ ) had similar efficacy in reducing EDS, and sodium oxybate ( $9 \mathrm{~g} / \mathrm{d}$ ) and pitolisant (21-40 $\mathrm{mg} / \mathrm{d}$ ) had a comparable beneficial effect for cataplexy (Figure 2).

The 2020 draft AASM guidelines* provide a strong recommendation for the use of pitolisant for narcolepsy.

Caffeine. Dr Roth discussed caffeine as an agent that may be useful to some patients with EDS. In a small study ${ }^{61}(\mathrm{~N}=16)$, researchers compared $200 \mathrm{mg}$ of caffeine with placebo in participants with narcolepsy; the first assessment followed the first dose, and the next assessment took place after 1 week of daily use. Results indicated that those taking the caffeine had significant improvements in alertness.

Behavioral strategies. Clinicians should discuss with patients the behavioral and lifestyle strategies that should be adopted to help manage narcolepsy, Dr Roth emphasized, including the following ${ }^{62}$ :

- Take short, regularly scheduled daytime naps. ${ }^{38,63,64}$

- Adhere to a consistent sleep-wake schedule.

- Exercise for at least 20 minutes per day at least 4 to 5 hours prior to bedtime.

- Maintain a comfortable, adequately cooled sleep environment.

- Engage in relaxing activities such as warm baths prior to bedtime.

- Avoid alcohol and caffeine for several hours prior to bedtime.

- Avoid smoking tobacco, especially at night.

- Take advantage of patient support groups. $\dagger$

The 2020 AASM guidelines* recommend that clinicians be aware that nonpharmacologic management strategies, besides good sleep hygiene and naps (eg, workplace or

\footnotetext{
*https://aasm.org/clinical-resources/practice-standards/ practice-guidelines/

†https://narcolepsynetwork.org/resources/support-groups/
}

Figure 2. Network Meta-Analysis Comparison of Modafinil, Pitolisant, and Sodium Oxybate in Individuals With Narcolepsy (14 Randomized Controlled Trials) ${ }^{a, b}$

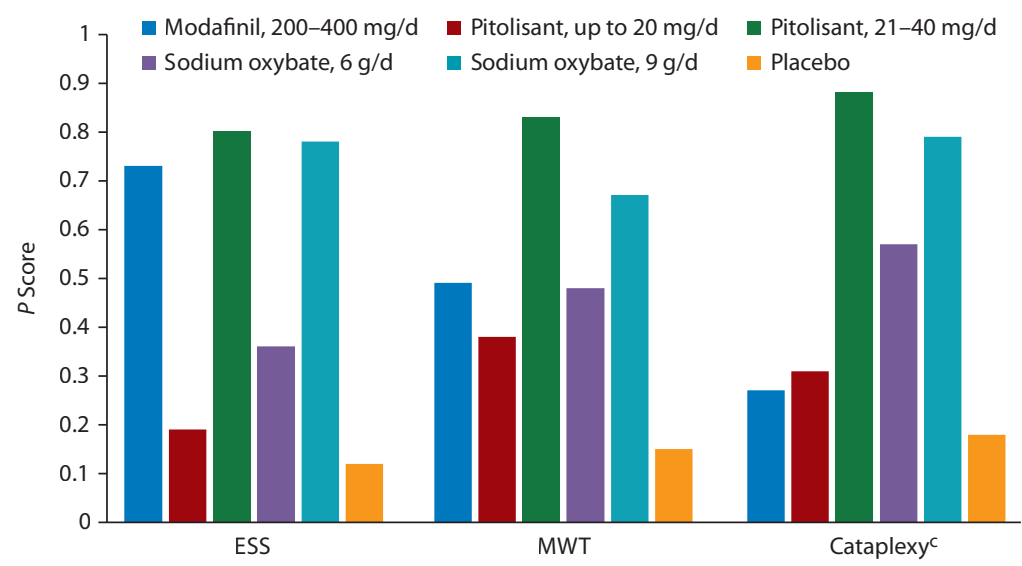

Outcome Measure

${ }^{a}$ Data from Lehert \& Falissard. ${ }^{59}$

bTreatment ranking by $P$ scores measures the extent of certainty that any one treatment is better than another treatment, averaged over all competing treatments (Rücker \& Schwarzer ${ }^{60}$ ).

${ }^{\mathrm{C} C}$ Cataplexy $=$ weekly rate of cataplexy attacks.

Abbreviations: ESS = Epworth Sleepiness Scale, MWT=Maintenance of Wakefulness Test.

educational disability accommodations, cognitive-behavioral therapy/ psychological support), are often needed to optimally treat patients regardless of drug treatments used.

\section{Case Practice Question}

Discussion of the best response can be found at the end of the activity.

Case 2. Alison, a 22-year-old university student, has been experiencing EDS since she was 15 years old. At her initial visit, her ESS score was 14, and she did not report any auxiliary symptoms. During the MSLT, she had 3 REM onsets on 5 nap opportunities and a mean sleep latency of 3.9 minutes. Alison was diagnosed with narcolepsy and treated with a stimulant medication and appropriate nonpharmacologic interventions. Significant improvement in her condition resulted from treatment. On a routine follow-up visit, she reports that in the past 6 months she has started to experience her knees buckling when she gets excited. While the events are not disabling, they are becoming more frequent and more severe. What next step should you take to manage Alison's cataplexy?

a. Avoid changing her treatment regimen, which has been working, until the cataplexy becomes a significant problem.

b. Add a daily anticataplexy medication to her stimulant.

c. Add an as-needed anticataplexy medication to her stimulant.

d. Try switching to a medication that is effective for both sleepiness and cataplexy.

\section{Ongoing Monitoring}

As patients receive treatment for narcolepsy, Dr Roth underscored that it is important for clinicians to provide ongoing monitoring of EDS so that they can adjust the regimen as needed to alleviate residual symptoms. A retrospective, longitudinal analysis ${ }^{65}$ of ESS scores for 26 patients with narcolepsy type 1 who received stable pharmacotherapy (mean duration, 5 years) found that $73 \%$ experienced improvement in sleepiness as indicated by a drop of 4 points or less from baseline. 
points) occurred in $15 \%$ of patients, while $12 \%$ of patients experienced worsening as indicated by an ESS score increase of 5 points. The 2020 AASM guidelines* recommend that clinicians regularly reassess treatment efficacy during follow-up visits so that treatment choices can be revised accordingly.

Dr Roth also discussed how pathophysiology informs emerging treatments. (For more information, see the activity "Treating Excessive Daytime Sleepiness in Patients With Narcolepsy" in this CME series.)

\section{Clinical Points}

- Evaluate the extent of patients' excessive daytime sleepiness using the Epworth Sleepiness Scale.

- Assign sleep diaries and/or actigraphy to collect data on patients' sleep habits.

- Use the results of the polysomnogram and Multiple Sleep Latency Test to confirm a diagnosis of narcolepsy.

- Consult treatment guidelines for up-to-date recommendations for narcolepsy.

- Counsel all patients with narcolepsy about implementing psychosocial strategies.

\section{Discussion of Case Practice Questions}

Case 1: Preferred response is c. Cholesterol level

Katie is taking 2 sedating medications, one of which is associated with significant weight gain, and these could cause her EDS. To evaluate the possibility of narcolepsy, she should be assessed for the presence of cataplexy, sleep paralysis, and hypnagogic hallucinations. Usual bedtime and waketime should be noted when patients have sleep problems. A high body mass index and the presence of snoring and witnessed apneas are associated with obstructive sleep apnea, ${ }^{66}$ but cholesterol level has little relation to daytime sleepiness.

Case 2: Preferred response is $\mathrm{d}$. Try switching to a medication that is effective for both sleepiness and cataplexy.

While previously Alison was being treated for essentially type 2 narcolepsy, it is now clear that she has type 1 narcolepsy and needs treatment for both her sleepiness and her cataplexy. As it is always preferable to use one medication rather than 2 , the best answer is $\mathrm{d}$.

Published online: November 24, 2020.

Disclosure of off-label usage: To the best of their knowledge, the authors have determined that caffeine and selegiline are not approved by the US Food and Drug Administration for the treatment of narcolepsy.

\section{REFERENCES}

1. Knie B, Mitra MT, Logishetty $K$, et al. Excessive daytime sleepiness in patients with Parkinson's disease. CNS Drugs. 2011;25(3):203-212.

2. Kolla BP, He J-P, Mansukhani MP, et al. Excessive sleepiness and associated symptoms in the US adult population: prevalence, correlates, and comorbidity. Sleep Health. 2020;6(1):79-87.

3. McWhirter D, Bae C, Budur K. The assessment, diagnosis, and treatment of

\footnotetext{
*https://aasm.org/clinical-resources/practice-standards/ practice-guidelines/
}

Psychiatry (Edgmont). 2007:4(9):26-35.

4. Pigeon WR, Sateia MJ, Ferguson RJ. Distinguishing between excessive daytime sleepiness and fatigue: toward improved detection and treatment. J Psychosom Res. 2003:54(1):61-69.

5. Slowik JM, Collen JF, Yow AG. Narcolepsy. In: StatPearls. Treasure Island, FL: StatPearls Publishing; 2020.

6. American Academy of Sleep Medicine. International Classification of Sleep Disorders. 3rd ed. Darien, IL: American Academy of Sleep Medicine; 2014.

7. Douglas NJ. The psychosocial aspects of narcolepsy. Neurology. 1998:50(suppl 1):S27-S30.

8. Overeem S, Black JL 3rd, Lammers GJ. Narcolepsy: immunological aspects. Sleep Med Rev. 2008;12(2):95-107.

9. Narcolepsy Fact Sheet. National Institute of Neurological Disorders and Stroke website. https://www.ninds.nih.gov/disorders/patient-caregivereducation/fact-sheets/narcolepsy-fact-sheet\#3201_4. Published March 16, 2020. Accessed May 1, 2020.

10. Maski K, Steinhart E, Williams D, et al. Listening to the patient voice in narcolepsy: diagnostic delay, disease burden, and treatment efficacy. J Clin Sleep Med. 2017;13(3):419-425.

11. Vasas A. Amanda's Story. Narcolepsy Network website. https:// narcolepsynetwork.org/amandas-story/. Accessed June 4, 2020

12. Plazzi G, Clawges HM, Owens JA. Clinical characteristics and burden of illness in pediatric patients with narcolepsy. Pediatr Neurol. 2018;85:21-32.

13. Daniels E, King MA, Smith IE, et al. Health-related quality of life in narcolepsy. J Sleep Res. 2001;10(1):75-81.

14. Teixeira VG, Faccenda JF, Douglas NJ. Functional status in patients with narcolepsy. Sleep Med. 2004;5(5):477-483.

15. Inocente CO, Gustin M-P, Lavault S, et al. Quality of life in children with narcolepsy. CNS Neurosci Ther. 2014;20(8):763-771.

16. Cohen A, Mandrekar J, St Louis EK, et al. Comorbidities in a community sample of narcolepsy. Sleep Med. 2018;43:14-18.

17. Ohayon MM. Narcolepsy is complicated by high medical and psychiatric comorbidities: a comparison with the general population. Sleep Med. 2013;14(6):488-492.

18. Ross H. What I Wish People Knew About Narcolepsy. Repeller website. https://repeller.com/narcolepsy-personal-experiences/. Published July 26, 2017. Accessed September 15, 2020.

19. Schmidt T. The Other Side of the Fence-Tesa's Story. Narcolepsy Network website. https://narcolepsynetwork.org/the-other-side-of-the-fence/. Accessed September 15, 2020.

20. Johns MW. Reliability and factor analysis of the Epworth Sleepiness Scale. Sleep. 1992;15(4):376-381.

21. Lawrence G, Muza R. Assessing the sleeping habits of patients in a sleep disorder centre: a review of sleep diary accuracy. J Thorac Dis. 2018;10(suppl 1):S177-S183.

22. Baumann CR, Mignot E, Lammers GJ, et al. Challenges in diagnosing narcolepsy without cataplexy: a consensus statement. Sleep. 2014:37(6):1035-1042.

23. Schwartz JR, Roth T, Hirshkowitz M, et al. Recognition and management of excessive sleepiness in the primary care setting. Prim Care Companion J Clin Psychiatry. 2009;11(5):197-204.

24. Morse AM. Narcolepsy in children and adults: a guide to improved recognition, diagnosis and management. Med Sci (Basel). 2019;7(12):106

25. Littner MR, Kushida C, Wise M, et al: Standards of Practice Committee of the American Academy of Sleep Medicine. Practice parameters for clinical use of the Multiple Sleep Latency Test and the Maintenance of Wakefulness Test. Sleep. 2005;28(1):113-121.

26. Narcolepsy: Testing. Healthy Sleep website. http://healthysleep.med harvard.edu/narcolepsy/diagnosing-narcolepsy/narcolepsy-testing. Published February 21, 2018. Accessed May 1, 2020.

27. Narcolepsy-Diagnosis. NHS UK website. https://www.nhs.uk/conditions/ narcolepsy/diagnosis/. Published May 13, 2019. Accessed May 1, 2020.

28. Mignot E, Lammers GJ, Ripley B, et al. The role of cerebrospinal fluid hypocretin measurement in the diagnosis of narcolepsy and other hypersomnias. Arch Neurol. 2002;59(10):1553-1562.

29. Heier MS, Evsiukova T, Vilming S, et al. CSF hypocretin-1 levels and clinica profiles in narcolepsy and idiopathic CNS hypersomnia in Norway. Sleep. 2007;30(8):969-973

30. Materna L, Halfter $\mathrm{H}$, Heidbreder A, et al. Idiopathic hypersomnia patients revealed longer circadian period length in peripheral skin fibroblasts. Front Neurol. 2018;9:424

31. Pérez-Carbonell L, Leschziner G. Clinical update on central hypersomnias. J Thorac Dis. 2018;10(suppl 1):S112-S123.

32. Higgins T. My story: living with narcolepsy. Virtual Mentor 2004;6(11):521-524.

33. Morgenthaler TI, Kapur VK, Brown T, et al; Standards of Practice Committee of the American Academy of Sleep Medicine. Practice parameters for the treatment of narcolepsy and other hypersomnias of central origin. Sleep. 2007:30(12):1705-1711.

34. OCEBM Levels of Evidence Working Group. OCEBM Levels of Evidence. Centre for Evidence-Based Medicine website. https://www.cebm. net/2016/05/ocebm-levels-of-evidence/. Published May 1, 2016. Accessed June 4, 2020.

35. Sackett DL. Rules of evidence and clinical recommendations for the 
'Tanagement of patients. Can J Cardiol. 1993;9(6):487-489.

36. Provigil—modafinil tablet [prescribing information]. DailyMed: US National Library of Medicine website. https://dailymed.nlm.nih.gov/ dailymed/druglnfo.cfm?setid=e16c26ad-7bc2-d155-3a5d-da83ad6492c8. Published March 2, 2020. Accessed July 13, 2020.

37. Nuvigil_armodafinil tablet [prescribing information]. DailyMed: US National Library of Medicine website. https://dailymed.nlm.nih.gov/ dailymed/druglnfo.cfm?setid=d878aed0-ddbf-8fa1-abf7-d3e480260845. Published March 2, 2020. Accessed July 14, 2020.

38. Littner M, Johnson SF, McCall WV, et al; Standards of Practice Committee. Practice parameters for the treatment of narcolepsy: an update for 2000. Sleep. 2001:24(4):451-466.

39. Billiard M, Besset A, Montplaisir J, et al. Modafinil: a double-blind multicentric study. Sleep. 1994;17(8 suppl):S107-S112.

40. Broughton RJ, Fleming JA, George CF, et al. Randomized, double-blind, placebo-controlled crossover trial of modafinil in the treatment of excessive daytime sleepiness in narcolepsy. Neurology. 1997:49(2):444-451.

41. US Modafinil in Narcolepsy Multicenter Study Group. Randomized trial of modafinil as a treatment for the excessive daytime somnolence of narcolepsy: Neurology. 2000;54(5):1166-1175

42. US Modafinil in Narcolepsy Multicenter Study Group. Randomized trial of modafinil for the treatment of pathological somnolence in narcolepsy. Ann Neurol. 1998;43(1):88-97.

43. Mitler MM, Harsh J, Hirshkowitz M, et al. Long-term efficacy and safety of modafinil (PROVIGIL((R))) for the treatment of excessive daytime sleepiness associated with narcolepsy. Sleep Med. 2000;1(3):231-243.

44. Schwartz JRL, Feldman NT, Bogan RK, et al. Dosing regimen effects of modafinil for improving daytime wakefulness in patients with narcolepsy. Clin Neuropharmacol. 2003;26(5):252-257.

45. Myrick H, Malcolm R, Taylor B, et al. Modafinil: preclinical, clinical, and post-marketing surveillance-a review of abuse liability issues. Ann Clin Psychiatry. 2004;16(2):101-109.

46. Billiard M, Bassetti C, Dauvilliers Y, et al; EFNS Task Force. EFNS guidelines on management of narcolepsy. Eur J Neurol. 2006;13(10):1035-1048.

47. Xyrem - sodium oxybate solution [prescribing information]. DailyMed: US National Library of Medicine website. https://dailymed.nIm.nih.gov/ dailymed/druglnfo.cfm?setid=926eb076-a4a8-45e4-91ef-411f0aa4f3ca. Published October 26, 2018. Accessed July 13, 2020.

48. Guilleminault C. Amphetamines and narcolepsy: use of the Stanford database. Sleep. 1993;16(3):199-201.

49. Parkes JD, Dahlitz M. Amphetamine prescription. Sleep. 1993;16(3):201-203.

50. Hublin $C$, Partinen $M$, Heinonen $E H$, et al. Selegiline in the treatment of narcolepsy. Neurology. 1994;44(11):2095-2101.

51. Mayer G, Ewert Meier K, Hephata K. Selegeline hydrochloride treatment in narcolepsy: a double-blind, placebo-controlled study. Clin Neuropharmacol. 1995;18(4):306-319.

52. Sunosi-solriamfetol tablet, film coated [prescribing information]. rightilted

.

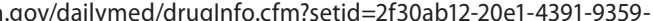
24b23a21578d. Published October 16, 2019. Accessed July 13, 2020.

53. Thorpy MJ, Shapiro C, Mayer G, et al. A randomized study of solriamfeto for excessive sleepiness in narcolepsy. Ann Neurol. 2019;85(3):359-370.

54. Carter LP, Henningfield JE, Wang YG, et al. A randomized, double-blind, placebo-controlled, crossover study to evaluate the human abuse liability of solriamfetol, a selective dopamine and norepinephrine reuptake inhibitor. J Psychopharmacol. 2018;32(12):1351-1361.

55. Wakix - pitolisant hydrochloride tablet, film coated [prescribing information]. DailyMed: US National Library of Medicine website. https:// dailymed.nIm.nih.gov/dailymed/druglnfo.cfm?setid=8daa5562-824e476c-9652-26ceef3d4b0e. Published November 6, 2019. Accessed July 13 2020.

56. Romigi A, Vitrani G, Lo Giudice T, et al. Profile of pitolisant in the management of narcolepsy: design, development, and place in therapy. Drug Des Devel Ther. 2018;12:2665-2675.

57. Szakacs Z, Dauvilliers Y, Mikhaylov V, et al; HARMONY-CTP study group. Safety and efficacy of pitolisant on cataplexy in patients with narcolepsy: a randomised, double-blind, placebo-controlled trial. Lancet Neurol. 2017;16(3):200-207.

58. Lehert P, Szoeke C. Comparison of modafinil and pitolisant in narcolepsy: a non-inferiority meta-analytical approach. Drugs Context. 2020;9:2020-6-2.

59. Lehert $P$, Falissard B. Multiple treatment comparison in narcolepsy: a network meta-analysis. Sleep. 2018:41(12):zsy185.

60. Rücker G, Schwarzer G. Ranking treatments in frequentist network metaanalysis works without resampling methods. BMCMed Res Methodol. 2015;15(1):58.

61. Aldosari MS, Olaish AH, Nashwan SZ, et al. The effects of caffeine on drowsiness in patients with narcolepsy: a double-blind randomized controlled pilot study [published online ahead of print March 26, 2020]. Sleep Breath.

62. What Treatments Are Available? Narcolepsy Fact Sheet. National Institute of Neurological Disorders and Stroke website. https://www.ninds.nih.gov/ Disorders/Patient-Caregiver-Education/Fact-Sheets/Narcolepsy-FactSheet\#3201_7. Published March 16, 2020. Accessed June 4, 2020.

63. Mullington J, Broughton R. Scheduled naps in the management of daytime sleepiness in narcolepsy-cataplexy. Sleep. 1993;16(5):444-456.

64. Rogers AE, Aldrich MS, Lin X. A comparison of three different sleep schedules for reducing daytime sleepiness in narcolepsy. Sleep. 2001;24(4):385-391.

65. Büchele F, Baumann CR, Poryazova R, et al. Remitting narcolepsy? longitudinal observations in a hypocretin-deficient cohort. Sleep. 2018:41(9).

66. Coutinho Costa J, Rebelo-Marques A, Machado JN, et al. Validation of NoSAS (Neck, Obesity, Snoring, Age, Sex) score as a screening tool for obstructive sleep apnea: analysis in a sleep clinic. Pulmonology. 2019;25(5):263-270.

\section{POSTTEST}

To obtain credit, go to PSYCHIATRIST.COM (Keyword: Text) to complete the Posttest and Evaluation.

1. The diagnostic feature that differentiates narcolepsy type 1 from type 2 is which of the following?

a. Total score of 10 or higher on the Epworth Sleepiness Scale

b. The presence of cataplexy

c. Mean sleep latency time of 8 minutes or less

d. Two or more onsets of rapid eye movement sleep during the Multiple Sleep Latency Test

2. Of the following medications, which one is not effective for cataplexy?

a. Sodium oxybate

b. Selegiline

c. Pitolisant

d. Modafinil

3. Kirk is a 22-year-old college student seeking help for excessive daytime sleepiness, which greatly impairs his ability to get his schoolwork done. You determine that Kirk is getting an adequate amount of time in bed and adequate sleep that is of adequate quality. All of the following tests are needed to make a diagnosis for Kirk except

a. Thyroid-stimulating hormone test

b. Polysomnogram

c. Multiple Sleep Latency Test

d. Epworth Sleepiness Scale

For reprints or permissions, contact permissions@psychiatrist.com. @ 2020 Copyright Physicians Postgraduate Press, Inc. 